\title{
Nucleolipoplexes: a new paradigm for phospholipid bilayer-nucleic acid interactions
}

\author{
Silvia Milani, Francesca Baldelli Bombelli, Debora Berti, Piero Baglioni
}

Department of Chemistry and CSGI, University of Florence, Via della Lastruccia 3- Sesto Fiorentino, 50019

Florence

\section{Supporting Information}

Materials: 1-Palmitoyl-2-oleoyl-sn-Glycero-3-Phosphocholine (POPC) was purchased from Avanti Polar Lipids (Alabaster, Alabama, USA) and its purity checked by TLC. The lecithin was used as received since no oxidation or lyso products could be detected. Adenosine, $\mathrm{HCl}, \mathrm{CHCl}_{3}$, $\mathrm{MeOH}$, and $\mathrm{NH}_{3}$ (33\% aqueous solution) used in the synthesis were purchased from Fluka (Switzerland). Phospholipase D from Streptomyces sp AA586 was a generous gift from Asahi Chemical Industry Co., Ltd (Tokyo, Japan). Tris buffer and polyUridylic acid potassium salt were purchased from Sigma-Aldrich.

POPA was synthesized starting from the corresponding phosphatidylcholine in a two-phase system according to a modification of the method proposed by Shuto and coworkers, and obtained as an ammonium salt. Separation from the by-products was achieved by silicagel flash chromatography. Purity was checked by TLC, ${ }^{1} \mathrm{H}$ NMR and elementary. Following, POPA ammonium salt was transform in sodium salt by titration. The chemical structure of polyU and POPA are reported in chart 1 .

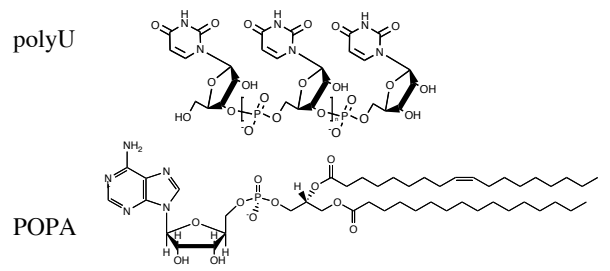

Chart 1. Schematic drawing of the chemical structures of PolyU and POPA. Uridine and Adenosine are a complementary pair in RNA.

Sample Preparation: The polynucleotide dissolved in TRIS buffer was added to $10 \mathrm{mg}$ of liponucleoside sodium salt to obtain the wanted POPA/PolyU ratio. The samples were homogenized by centrifugation and several cycles of freeze/thaw. Samples have been measured after preparation and after an annealing treatment. The annealing procedure consists of heating the samples at $50^{\circ} \mathrm{C}$ and equilibrating them at $4^{\circ} \mathrm{C}$ for about a week. All the samples have been checked several times over months and they were stable.

Small angle X-ray diffraction (SAXD): SAXD experiments were performed on SAXS-WAXS Hecus X-ray system GMBH (Graz, Austria) containing 1024 channels. The working q-range was $0.05-6 \mathrm{~nm}^{-}$ ${ }_{1}$ corresponding to Bragg's spacing of about 120 to $1 \mathrm{~nm}$. $\mathrm{Cu} \mathrm{K \alpha}$ radiation of wavelength $1.542 \AA$ was provided by a Seifert X-ray generator operated at $40 \mathrm{KV} 10 \mathrm{~mA}$. The samples were mounted in a kapton sample holder. To minimize the scattering from air, the camera volume was kept under vacuum during the measurements. Temperature control within $0.1^{\circ} \mathrm{C}$ was achieved by using a Peltier element.
POPA:polyA bilayers: SAXD measurements of POPA:polyadenilic acid (polyA) (2:1) are reported in Figure 1 Supporting Information. POPA bilayers do not display any modifications of the bilayer thickness when interacting with non-complementary polynucleotide as found for the POPA/PolyU ( see main text ). The samples were prepared in the same way as the POPA/PolyU ones.

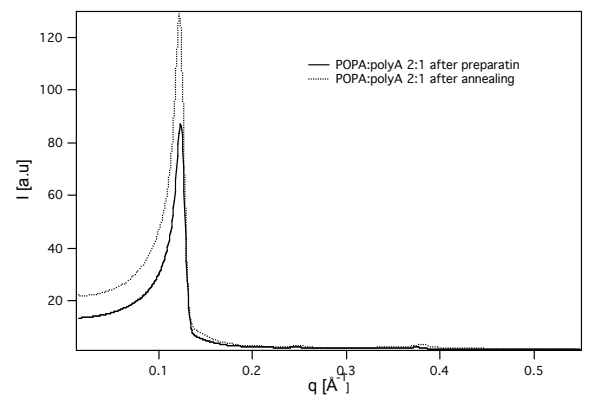

Figure 1_Supporting Information. Small Angle X-ray Diffraction Spectra of POPA:polyA (2:1) bilayers.

Fourier Transform Infrared Spectroscopy (FTIR): IR spectra were collected with a Nexus 870 spectrophotometer (Thermo Nicolet, Paris.) equipped with a pyroeletric detector (DTGS-TEC). The samples were squeezed in two $\mathrm{CaF}_{2}$ windows. All spectra were performed at room temperature with $2 \mathrm{~cm}^{-1}$ resolution and averaging 250 scans.

IR de-convolution: the absorption between $1800-1550 \mathrm{~cm}^{-1}$ is due to several vibrations and it was de-convolved using Gaussians contributions. The number of fitting functions was chosen in agreement to the conventional literature both for the lipid ${ }^{1}$ and nucleic acids ${ }^{2,3}$. Figure 2 supporting Information and Table1 Supporting Information show the de-convoluted absorptions of pure POPA bilayers at $50 \% \mathrm{w} / \mathrm{w}$ Tris buffer and the attribution of the calculated frequencies. These values together with those characteristic for polyU have been used to de-convolve POPA:polyU spectra in the region between $1800-1550 \mathrm{~cm}^{-1}$. Nine functions were used to de-convolve these spectra. Indeed, the absorptions between $1800-1550 \mathrm{~cm}^{-1}$ is composed of nine contributions; five are from POPA (see table 1 supporting information) and four from polyU ${ }^{3}$. Figure 3 and 4_supporting Information and Table2 and 3 Supporting Information point out that the most important difference between POPA:polyU FTIR spectra freshly prepared and after the annealing treatment is the shift of the $\mathrm{C}=\mathrm{O}$ stretching of uridine base when the nucleolipoplex complex is formed. These shifts are in agreement to the formation of a Watson-Crick base pair ${ }^{4}$. 


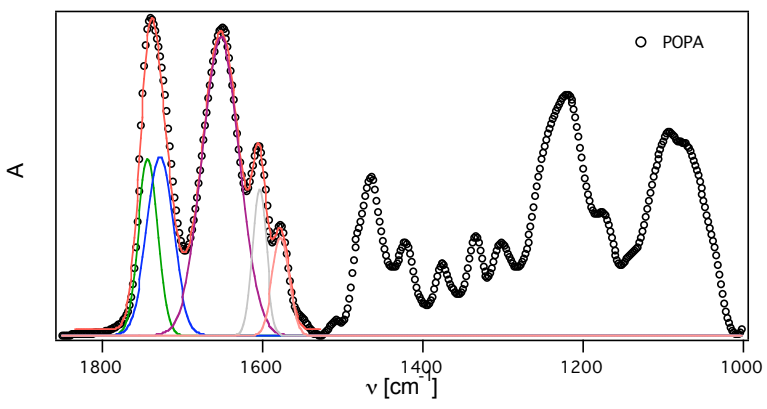

Figure 2_Supporting Information. FTIR spectrum of pure POPA bilayers. In red is reported the fitting curve; green, blue, purple, gray and pink are the de-convolved bands by means of five Gaussians. $\mathrm{A}=$ Absorbance.

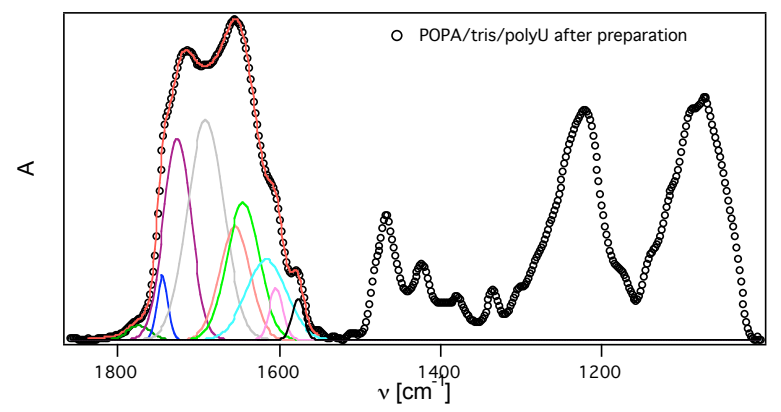

Figure 3_Supporting Information. Absorbance spectrum of POPA: polyU 2:1 bilayers right away after preparation. In red is reported the fitting; green, blue, purple, gray, pink, cyan, magenta and black are the de-convolved bands by means of Gaussians. A=Absorbance.

Table 1_supporting Information

\begin{tabular}{c|ccc}
$\begin{array}{l}\text { POPA Tris } \\
\mathbf{5 0 \%}[\mathbf{w} / \mathbf{w}]\end{array}$ & curve colors & $\boldsymbol{v}\left[\mathbf{c m}^{-1}\right]$ & attribution \\
\hline & green & $1743 \pm 1$ & $\mathrm{C}=\mathrm{O}$ \\
& blue & $1728 \pm 1$ & $\mathrm{C}=\mathrm{O}$ \\
& purple & $1652 \pm 1$ & $\delta \mathrm{NH}_{2}+v$ ring \\
& grey & $1604 \pm 1$ & $v$ ring $+\delta \mathrm{NH}_{2}$ \\
& pink & $1577 \pm 1$ & $v$ ring in plane
\end{tabular}

Table 2_Supporting Information

\begin{tabular}{c|ccc}
$\begin{array}{c}\text { POPA: polyU } \\
\text { 2:1 Tris 50\% } \\
\text { [w/w] after } \\
\text { preparation }\end{array}$ & curve color & $\boldsymbol{v}\left[\mathbf{c m}^{-1}\right]$ & attribution \\
\hline & & & \\
& green & $1772 \pm 1$ & \\
blue & $1745 \pm 1$ & $\mathrm{C}=\mathrm{O}$ \\
& purple & $1728 \pm 1$ & $\mathrm{C}=\mathrm{O}$ \\
& grey & $1691 \pm 1$ & $v \mathrm{C} 2=\mathrm{O}$ polyU \\
& pink & $1660 \pm 1$ & $v \mathrm{C} 4=\mathrm{O}$ polyU \\
& light green & $1651 \pm 1$ & $\delta \mathrm{NH}_{2}+v$ ring \\
& cyan & $1617 \pm 1$ & $v$ ring polyU
\end{tabular}

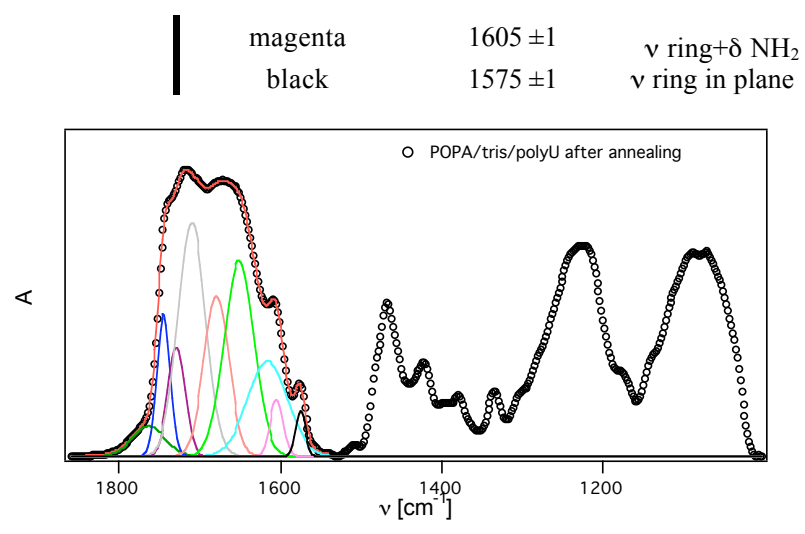

Figure 4_Supporting Information. FTIR spectrum of POPA:polyU (2:1) bilayers after annealing treatment. In red is reported the fitting curve; green, blue, purple, gray, pink, cyan, magenta and black are the deconvolved bands by means of Gaussians. A=Absorbance..

Table 3_Supporting Information

\begin{tabular}{|c|c|c|c|}
\hline $\begin{array}{l}\text { POPA: polyU } \\
\text { 2:1 Tris } 50 \% \\
\text { [w/w] after } \\
\text { annealing }\end{array}$ & curve color & $v\left[\mathrm{~cm}^{-1}\right]$ & attribution \\
\hline & $\begin{array}{l}\text { green } \\
\text { blue } \\
\text { purple } \\
\text { grey }\end{array}$ & $\begin{array}{l}1773 \pm 1 \\
1745 \pm 1 \\
1729 \pm 1 \\
1712 \pm 1\end{array}$ & $\begin{array}{c}\mathrm{C}=\mathrm{O} \\
\mathrm{C}=\mathrm{O} \\
\vee \mathrm{C} 2=\mathrm{O} \text { polyU }\end{array}$ \\
\hline & pink & $1675 \pm 1$ & $v \mathrm{C} 4=\mathrm{O}$ poly $\mathrm{U}$ \\
\hline & $\begin{array}{l}\text { light green } \\
\text { cyan }\end{array}$ & $\begin{array}{l}1655 \pm 1 \\
1617 \pm 1\end{array}$ & $\begin{array}{c}\delta \mathrm{NH}_{2}+v \text { ring } \\
v \text { ring polyU }\end{array}$ \\
\hline & $\begin{array}{l}\text { magenta } \\
\text { black }\end{array}$ & $\begin{array}{l}1604 \pm 1 \\
1576 \pm 1\end{array}$ & $\begin{array}{c}v \text { ring }+\delta \mathrm{NH}_{2} \\
v \text { ring in plane }\end{array}$ \\
\hline
\end{tabular}

UV-spectroscopy: UV-vis spectra were recorded on a double beam Perkin-Elmer Lambda 5 spectrometer. Hellma cells with a $0.01 \mathrm{~mm}$ path were used for the measurements. Since the samples are highly concentrated, an internal standard (retinol) was used to compare the spectra obtained on the same sample at different temperature and after the annealing treatment. Since retinol is a hydrophobic molecule, it is embedded into the membrane. The advamtage in using it is mainly related to the absorbance peak between 300 and $400 \mathrm{~nm}$ that does not strongly affect the main absorbance peak at $260 \mathrm{~nm}$ of the nucleolipid and the polynucleotide. Moreover, to take into account possible modifications of the spectra due to scattering or environment differences, we have compared the retinol absorption peaks. However, to reduce these differences we have performed UV measurements on the same sample. Figure 5_Supporting Information shows that the characteristic base peak at $260 \mathrm{~nm}$ decreases upon the formation of the nucleolipoplex complex. This hypochromic effect is ascribable to stacking excess due to the formation of A-U base pair. On the other hand, the hyperchromic effect observed upon the heating to $50^{\circ} \mathrm{C}$ is related to a known de-stacking effect between neighbor adenosine bases on the membrane induced by the temperature increase. 


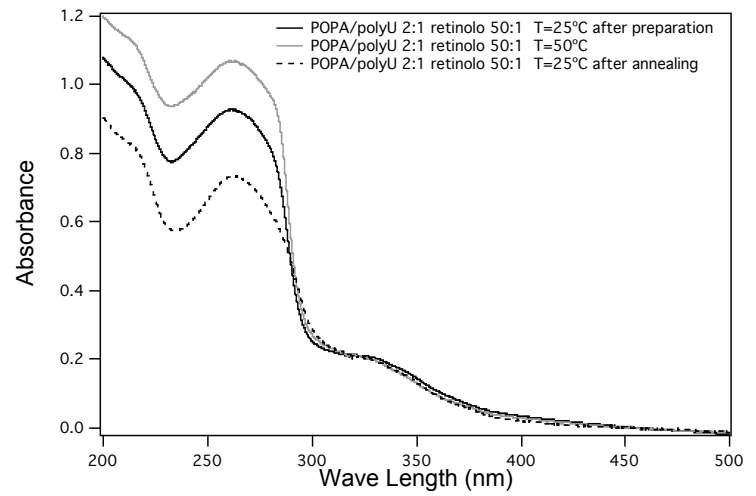

Figure 5_Supporting Information. UV spectra for POPA:polyU $(2: 1)$ bilayers sandwiched between sealed quartz slides. Black line freshly prepared at RT; grey line at $50^{\circ} \mathrm{C}$; dotted line at RT after the annealing treatment.

\section{REFERENCES}

(1) Fringeli, U. P.; Gunthard, H. H. In Membrane Spectrosocpy; Grell, E., Ed.; Springer-Verlag: Berlin, 1981.

(2) Banyay, M.; Sarkar, M.; Graslund, A. Biophys. Chem. 2003, 104, 477-488.

(3) Liquier, J.; Taillanddier, E. In Infrared spectroscopy of Biomolecules; Chapman, D., Ed.; Wiley-Liss Inc.: New York, 1996, pp 131-158.

(4) Miles, H. T.; Frazier, J. Biochemistry 1978, 17, 2920-2927. 